\title{
Versorgungsforschung - das Orchester ist wichtiger als das Instrument
}

\author{
Rosemann, Thomas
}

DOI: https://doi.org/10.1024/1661-8157/a002627

Posted at the Zurich Open Repository and Archive, University of Zurich ZORA URL: https://doi.org/10.5167/uzh-137837

Journal Article

Originally published at:

Rosemann, Thomas (2017). Versorgungsforschung - das Orchester ist wichtiger als das Instrument. Praxis, 106(7):339-340.

DOI: https://doi.org/10.1024/1661-8157/a002627 


\title{
Versorgungsforschung - das Orchester ist wichtiger als das Instrument
}

\author{
Thomas Rosemann
}

Dieses Heft widmet sich dem Schwerpunktthema «Versorgungsforschung». Ein Begriff, auf den Sie in den letzten Jahren wahrscheinlich häufiger gestossen sind. Was aber hat es damit auf sich? Ist das nur eine neue «Modeströmung» oder etwas, wovon unser Gesundheitssystem wirklich profitiert?

Dazu muss man sich einmal genauer betrachten, was Versorgungsforschung überhaupt erforscht. Der Sinn der Grundlagenforschung, wo es darum geht, im Labor neue Moleküle zu entdecken, aus denen einmal wirksame Medikamente werden, erschliesst sich jedem. Nachdem diese Moleküle im Labor entwickelt wurden, folgen klinische Studien. Hier geht es darum, die grundsätzliche Wirksamkeit von definierten Substanzen unter kontrollierten Bedingungen an meist hoch selektiertem Patienten zu untersuchen. Wenn auch diese Studien erfolgreich abgeschlossen wurden und die Zulassung erfolgt ist, dann erst beginnt der Einsatz unter Altagsbedingungen. Im Alltag können aber Medikamente - oder auch alle anderen therapeutischen oder diagnostischen Verfahren - ihren Nutzen nur entfalten, wenn sie adäquat eingesetzt werden. Adäquat bedeutet, beim «richtigen» Patienten zum «richtigen» Zeitpunkt eingesetzt werden. Ansonsten droht Unter- (falls sie überhaupt nicht oder zu wenig eingesetzt werden) oder Überversorgung (falls sie bei den Patienten eingesetzt werden, die nicht davon profitieren).

Was aber der «richtige» Patient» und der «richtige» Zeitpunkt ist, ist aus den klinischen Studien mit ihren Idealbedingungen und ihren hochselektierten Patienten nicht so einfach ableitbar, denn zwei grundsätzliche Fragen bleiben nach den meisten klinischen Studien offen: 1. sind die Ergebnisse auf den «alltäglichen» Patienten, der oft deutlich älter als der Studienpatient ist, und zudem relevante Begleiterkrankungen hat, überhaupt übertragbar? So zeigte beispielsweise eine Arbeit von Boyd et al (1), das in über $40 \%$ aller klinischen Diabetes-Trials Patienten mit Niereninsuffizienz oder einer KHK ausgeschlossen wurden. In klinischen Studien zur Herzinsuffizienz wurden in über $40 \%$ eine $\mathrm{KHK}$ und in fast $30 \%$ ein Hypertonus ausgeschlossen. Da fällt schon fast nicht mehr ins Gewicht, dass diese Patienten im Schnitt 20 Jahre jünger waren als der typische Patient mit dieser Grunderkrankung in der Hausarztpraxis. Da diese klinischen Studien, die Grundlage für Leitlinien bilden, haben sie dennoch erheblichen Einfluss auf die (scheinbare) Evidenz, an der wir uns orientieren müssen. Die zweite Frage ist, ob die diagnostischen oder therapeutischen Ansätze auch unter Alltagsbedingungen wirksam sind.

Versorgungsforschung erfasst daher auch Aspekte der Versorgung, die sich ebenfalls aus klinischen Studien nicht ableiten lassen: das Setting. Während in klinischen Studien das Setting möglichst standardisiert sein muss, um eben keinen Einfluss auf den Therapieeffekt zu haben, ist die Situation in der Realität eine ganz andere. Hier kommen kontextuelle Aspekte auf der Patientenebene, also beispielsweise die unmittelbaren Lebensumstände, aber vor allem auch auf 
Systemebene hinzu, die ganz wesentlich für den Erfolg einer Diagnostik oder Therapie sind.

So ist unser Gesundheitssystem heute gekennzeichnet von einer zunehmenden Spezialisierung und Fragmentierung, was wiederrum mit zahlreichen

Schnittstellenproblemen einhergeht. Je grösser aber quasi das «Orchester» der medizinischen Versorgung wird, desto besser müssen die einzelnen Musiker und Instrumente aufeinander abgestimmt werden. Das Instrument an sich, also etwa das Medikament, das in der klinischen Studie im Mittelpunkt steht, spielt dabei fast schon eine untergeordnete Rolle. Versorgungsforschung ist somit weniger am einzelnen Ton eines einzelnen Medikamentes interessiert, sondern am gesamten Klangbild des Orchesters.

Versorgungsforschung wird manchmal als rein deskriptives Fach missverstanden, das nur Missstände aufzeigen würden. Das ist so nicht richtig. Natürlich sind eine valide Beschreibung der Ausgangssituation und die Analyse möglicher Lösungsansätze die Voraussetzung. Aber randomisierte Studien gehören zum festen Repertoire der Versorgungsforschung: unter real-life Bedingungen testet sie Ansätze, oft sogenannte «multi-faceted interventions», also Interventionen, die unterschiedliche Elemente enthalten, um die Versorgung effizienter zu gestalten.

Etwas vereinfacht ausgedrückt könnte man also sagen, Versorgungsforschung bemüht sich darum, das richtige Mass an Medizin zum richtigen Zeitpunkt an den richtigen Patienten zu bringen. Anstatt der Wirksamkeit unter kontrollierten Bedingungen, in kontrollierten Settings, eben der «Efficacy», geht es ihr um die Wirksamkeit unter Alltagsbedingungen unter unkontrollierten und unselektierten Bedingungen. Im Fokus stehen dabei vor allem patientenrelevante, klinische Outcomes. Man spricht von der «Effectiveness».

Versorgungsforschung ist somit quasi die Grundvoraussetzung für ein Gesundheitssystem, das sich den realen und tatsächlichen Bedürfnissen des Patienten auszurichtet. Auch im Schweizer Gesundheitssystem sind die monetären wie personellen Ressourcen nicht unbegrenzt verfügbar. Eine zukunftsfähige Ausrichtung kann nicht auf Partikularinteressen und daraus resultierender Willkür beruhen, daher brauchen auch wir eine evidenzbasierte Grundlage für Entscheidungen über eine effiziente Gestaltung und Massnahmen - auf der Praxisoder Spitalsebene ebenso wie im System als Ganzes.

In diesem Schwerpunktheft erhalten Sie einen interessanten Einblick über die methodische wie inhaltliche Vielfalt der Versorgungsforschung.

Versorgungsforschung ist übrigens wie die Demokratie: sie braucht oftmals auch aktives Mitwirken. Ich hoffe, dieses Heft weckt Ihr Interesse einmal an einem Forschungsprojekt, das genau Ihren Tätigkeitsbereich adressiert, mitzuwirken. So können auch Sie mit erforschen, was im Alltag «funktioniert» und was nicht und damit Ihren Beitrag für ein zukunftsfähiges Gesundheitssystem leisten.

(1) Boyd CM et al. PLoS One. 2012;7(8):e41601 